\title{
A NEW CONTROL STRATEGY FOR A SEMI-ACTIVE DIFFERENTIAL (PART II)
}

\author{
Ferruccio Resta ${ }^{1}$, Gerald Teuschl ${ }^{2}$, Mauro Zanchetta ${ }^{3}$, Andrea Zorzutti ${ }^{1}$. \\ ${ }^{1}$ Politecnico di Milano \\ Via La Masa 34, 20156 Milano, Italy \\ ${ }^{2}$ Elektrik Elektronik Engineering \\ MAGNA STEYR Fahrzeugtechnik AG \& Co KG, \\ Liebenauer Hauptstrasse 317, A-8041 Graz, Austria \\ ${ }^{3}$ Ferrari S.p.A. - Gestione Ind. \\ Via Abetone Inferiore 4, 41053 Maranello, Modena, Italy
}

\begin{abstract}
VDC systems usually operate on the engine torque and on brake pressures, new automotive applications try to use semi-active or active differentials in order to optimize the torque distribution on the wheels for traction maximization, driving comfort, stability and safety of the vehicle. The system presented in the paper comes out from the cooperation of Ferrari, MAGNA STEYR and Politecnico di Milano in the development of a semi-active differential. In the paper a description of either the vehicle dynamic control strategy, its development, and of the experimental results following its implementation on a existent vehicle are given. Copyright $($ C 2005 IFAC
\end{abstract}

Keywords: Differential, Vehicle, Control, Software, Logic, Automotive.

\section{INTRODUCTION}

Every car needs a differential, which is a mechanism that lets the driven wheels to assume different speeds while vehicle is in curve. The difference of wheel speeds comes from kinematical considerations and it can't be neglected. On common cars a free differential is usually mounted. This device is a simple gearing that always guarantees an equal driving torque distribution. Free differential is a simple and cheap device, but on the other hand it decreases the traction performance because it cannot transfer driving torque to the wheel on higher adherence or to the more loaded one. This lack has been mechanically approached in different ways: the most common one foresees a clutch that contrasts the relative motion of the driven shafts. This kind of device, usually called torque-sensitive differential, often improves car traction optimising the torque distribution, but it introduces an uncomfortable under/oversteering behaviour. Self-locking differential influence, in steady state condition, has been explained in the first part of this paper (Cheli, et al., 2005), but this is not the only case in which this device negatively compromises vehicle handling.

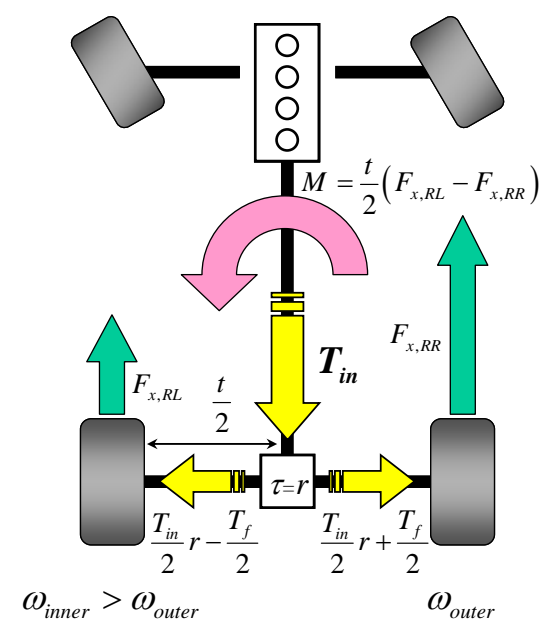

Fig. 1. Self-locking differential: oversteering moment (power on). 
When the vehicle is in power on turning condition (Fig. 1), that is when the driver pushes the throttle in a turn, the self-locking differential transfers torque to the slowest wheel (the outer one), generating an oversteering moment up to vehicle spin.

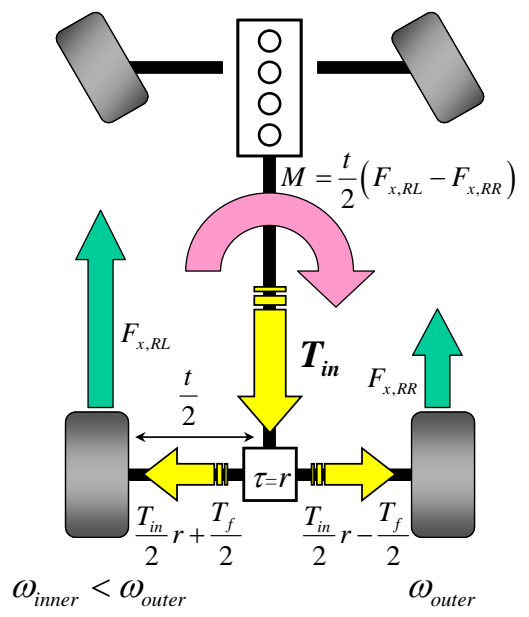

Fig. 2. Self-locking differential: understeering moment (power off).

Another critical condition for car stability is the power off turning condition, that is when the throttle is released and the consequent load transfer reduces rear axle adherence. In this case a free differential, causes path saturation of the outer wheel and, consequently, the vehicle spin. In the same condition a self-locking differential transfers more driving torque to the inner wheel (which would spin slower), generating an understeering moment (Fig. 2). Since, in this way, the outer wheel path is not saturated the vehicle is stable and it doesn't spin.

It can be easily understood that a controlled torque transfer can optimize vehicle performance in every condition, while a passive differential can only reach an acceptable compromise.

\section{CONTROL LOGIC DESIGN METHOD}

As introduced in the first part of this paper (Cheli, et al., 2005), considering the different reaction of the car to the locking torque, a vehicle dynamic control logic based on feed-forward and feed-back components has been designed and implemented. The approach adopted to define the reference functions of the feed-forward components was based on an intensive numerical simulation campaign. Due to this fact, performance of feed-forward components strongly depends on the reliability of the vehicle model (see section 3). For this reason it was used a validated 14 degrees of freedom model (IPG CarMaker ${ }^{\circledR}$ ) of the vehicle, integrated with the control logic and the actuation model. Once the logic was developed, it was tested on real vehicle and system parameters were tuned (Fig. 3).

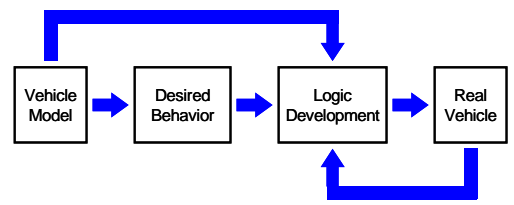

Fig. 3. Logic development approach.

In section 3 a short description of vehicle model validation is given. In section 4 main logic architecture is explained. In sections 5, 6 and 7 a detailed description of the whole algorithms is given.

\section{VEHICLE MODEL VALIDATION}

A 14 degrees of freedom vehicle model (IPG CarMaker $^{\circledR}$ ) was used off-line to analyse car behaviour in several conditions, to define a strategy for each one and to get a first tuning of the whole system. In the model the elasto-kinematics of the suspensions is described with multidimensional lookup tables obtained from analyses run with a multi body complex model (ADAMS/Car ${ }^{\circledR}$ ). The Pacejka combined model has been used to describe tyre be-haviour. In this way it was possible to reproduce car behaviour even at limit, where the nonlinearities are not negligible. The model has been validated comparing its outputs to experimental data measured on a demo target vehicle. As an example in Fig. 4 and Fig. 5 some result of validation process are plotted. In Fig. 4 the understeer curve (Steer angle vs. $A_{Y}$ ) of a constant radius steering pad manoeuvre is shown.

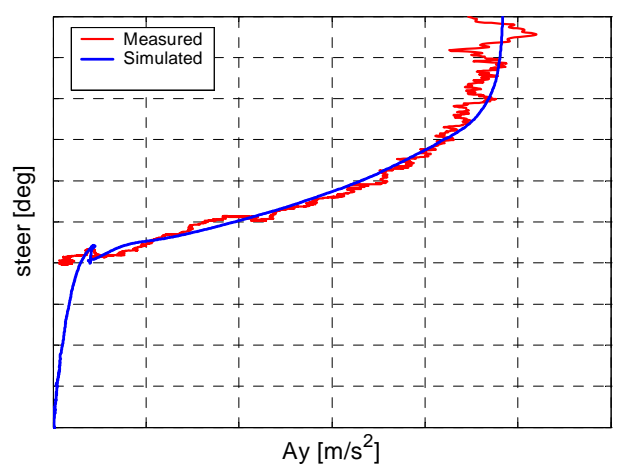

Fig. 4. Steering pad constant radius. Understeering curve numerical vs. experimental.

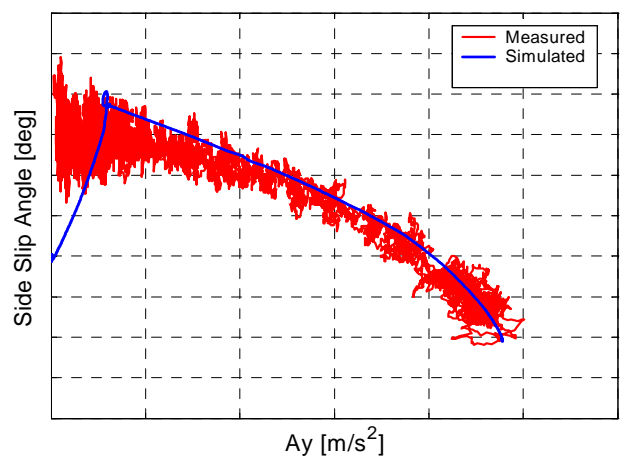

Fig. 5. Steering pad constant radius. Side slip curve numerical vs. experimental. 
In Fig. 5 the car sideslip curve (Car Side Slip vs. $A_{Y}$ ) of the same manoeuvre is shown. It's important to observe a satisfactory matching between numerical result and experimental data.

\section{LOGIC MAIN ARCHITECTURE}

In section 1 it has been underlined that differential influence closely depends on driver demands and driving torque sign: that means it is different in power on and power off condition. Even if the goal is always to maintain car stability also at vehicle limit, the approach to achieve it is different in steadystate/power on and power off situations. In the first case a free differential is preferred, in the other case a locked one is preferred. This distinction is a basic idea in control logic too (Fig. 6). First of all the algorithm foresees that car state has to be detected. The system, then, applies dedicated sub-algorithms, one for steady-state/power on and one for power off, which results represent the reference locking torque $T_{f, r e f}$. As previously explained both algorithms define $T_{f, r e f}$ as a sum of a feed-forward (consisting in multidimensional maps) and a close-loop component. This approach is very useful because measured signals (lateral acceleration, yaw rate, etc.), commonly used in vehicle control systems, are consequences of driver inputs and are affected by time delays due to vehicle dynamics. These delays practically reduce control bandwidth. The approach adopted avoids this problem: the feed-forward component guarantees a quick system response, and the feed-back one guarantees a precise control.

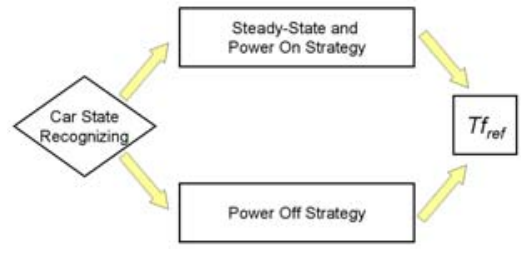

Fig. 6. Logic main architecture.

\section{STEADY-STATE AND POWER ON}

Steady-state and power on strategy is based on speed difference $\Delta V_{X, i s t}$ between inner wheel and outer one. It is defined as:

$$
\Delta V_{X, i s t}=\left(\omega_{1}-\omega_{2}\right) \cdot r_{\text {stat }} \cdot \operatorname{sign}(\dot{\psi})
$$

in which $\omega_{1}$ and $\omega_{2}$ are wheel rotational speeds [rad/s], $r_{\text {stat }}$ is the wheel static radius [m], and $\dot{\psi}$ is the yaw rate $[\mathrm{rad} / \mathrm{s}]$. This value is compared with a threshold $\Delta V_{x, \text { threshold }}$ coming from a function of vehicle state (2), whose values are defined by numerical and experimental tuning campaigns.

$$
\Delta V_{X, \text { threshold }}=f\left(V_{X}, A_{Y}, \dot{\psi}, T_{\text {Engine }}\right)
$$

The difference between $\Delta V_{x, \text { ist }}$ and $\Delta V_{x, \text { threshold }}$ is the error $\varepsilon$, which is used by a PI controller to calculate the reference locking torque $T_{f, \text { ref }}$. The PI controller gains are non-linear. Threshold $\Delta V_{x, \text { threshold }}$ is constituted by two components (Fig. 7):

- $\quad$ steady-state component;

- power on component, which is added to steady-state one only when a power on transient manoeuvre is recognized.

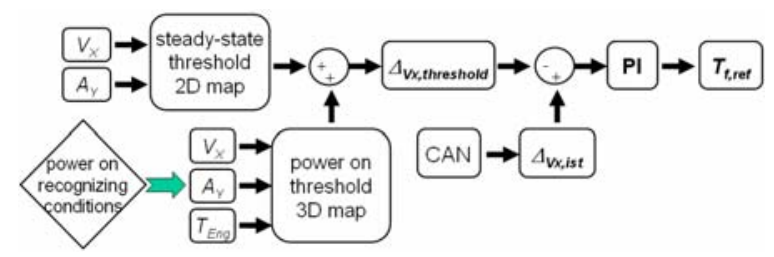

Fig. 7. $\Delta V_{X \text {,threshold }}$ and $T_{f, \text { ref }}$ definition.

The procedure followed for the definition of the steady-state component of the threshold foresees the following steps:

- simulate the understeering curve at constant speed with controlled differential, setting different constant thresholds;

- define a desiderated understeering curve (Fig. 8) for each longitudinal speed;

- define a mathematical surface that can reproduce designed points.

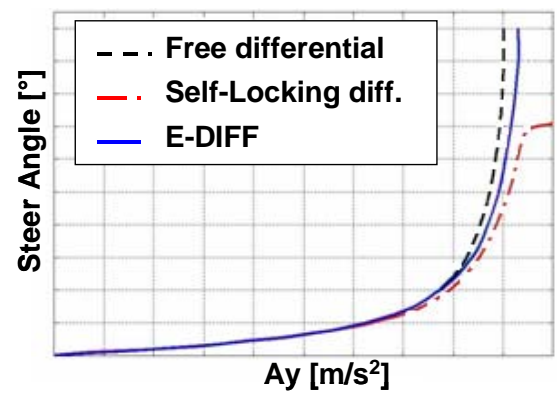

Fig. 8. Steady-state threshold definition procedure. Understeering curve at fixed speed.

In power on conditions wheel speeds difference would be greater than in steady-state conditions due to the higher driving torque. In these cases steadystate threshold would be too small and controlled differential would transfer too much torque to the outer wheel, generating an unpleasant and dangerous oversteer. This behaviour can be avoided increasing steady-state threshold by a power on additional value, function of speed, lateral acceleration and driving torque (Fig. 7). It's clear that with this approach the car doesn't reach the best performance in terms of traction, but the gain in terms of stability and driving feeling increases performance. The procedure adopted for the additional threshold definition was:

- simulate power on cornering from a steadystate initial condition at different initial speeds and with different torque demands;

- define the best additional threshold for each speed, lateral acceleration and driving torque combination and fit them with a mathematical surface. 


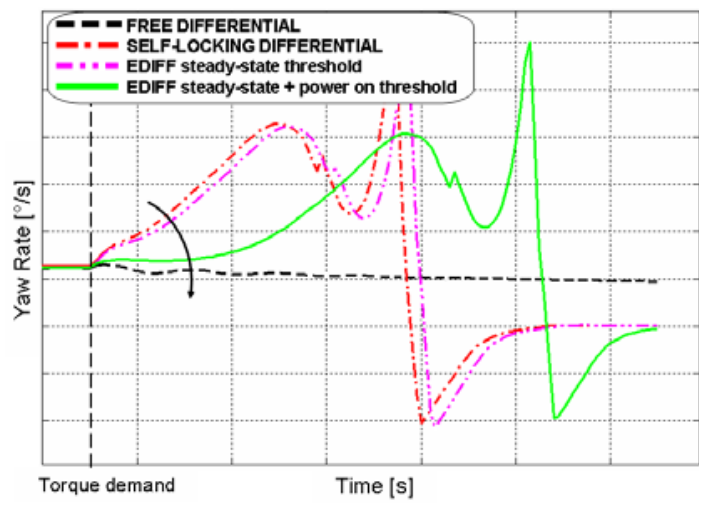

Fig. 9. Yaw rate with different differentials after torque demand.

The adopted criterion is based on a desired yaw rate behaviour or trend. In particular no vehicle spin due to power on is tolerated, in other words yaw rate must not increase in the very first time after the throttle step (Fig. 9). In Fig. 9 the yaw rate time histories referring to a power on in curve manoeuvre are reported. The driver pushes gas pedal at the time called "Torque demand". Power on additional threshold, that makes controlled differential behaviour more similar to the free one, must be kept for a limited time and it has to end when it's not required for vehicle stability any more. It's also fundamental for the vehicle to keep a smooth behaviour while it's still turning. Because of that power on threshold ending condition has been analysed and developed with great care.

\section{POWER OFF}

A power off manoeuvre is really critical both from the vehicle dynamics point of view and from safety one. When car is close to the adherence limits, in other words at high lateral acceleration, the load transfer to the front axle, due to the power off, generates an oversteering moment. Even if car doesn't spin, its yaw rate suddenly increases and its path gets into a closer trajectory. This, at least, gives the driver an instability feeling. Therefore a locking differential can be helpful because it introduces an understeering moment on vehicle, which opposes its natural oversteering tendency. Because of that a controlled differential should increase the locking torque while car is approaching to its limit. In power off control strategy the locking torque is defined in feed-forward as a function of vehicle speed and lateral acceleration (Fig. 10). A dedicated power off feed-forward algorithm is necessary because a feedback strategy based on the measurements would react to the power off too slowly. There would be a time gap when controlled differential would behave as a free one, not introducing any understeering moment and not opposing to oversteering car tendency. An open loop strategy, instead, can order locking torque as soon as a power off condition is detected. Power off starting and ending condition are detected observing the driver input, with particular attention to the torque demand.

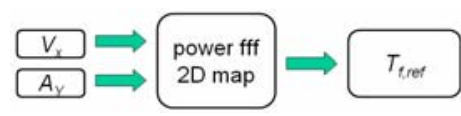

Fig. 10. Power off open loop strategy.

\section{PARTICULAR CONDITIONS: $\mu$-SPLIT}

The system implemented foresees some subsystem dedicated to particular driving conditions such as start on $\mu$-split. In this condition one driven wheel is on a low adherence (low $\mu$ ) surface, such as ice or mud, and it is not able to transmit the driving torque to the ground. A free differential would allow the wheel on low $\mu$ to spin, dissipating the whole driving power while the car doesn't move (Fig. 11).

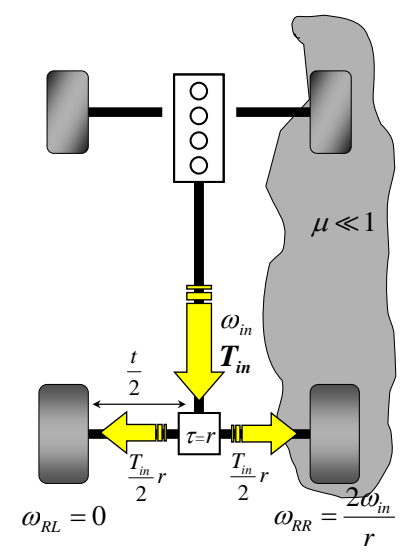

Fig. 11. Free differential in $\mu$-split condition.

A locking action of the differential transfers torque to the wheel on high $\mu$, improving traction, but, of course, it generates an oversteering moment on the vehicle, due to the not uniform distribution of longitudinal contact forces, (Fig. 12) that the driver has to contrast acting on the steering wheel. A good control system has to transfer to the high- $\mu$ wheel the right amount of torque, in order to reach a good compromise between longitudinal performance and driving feeling.

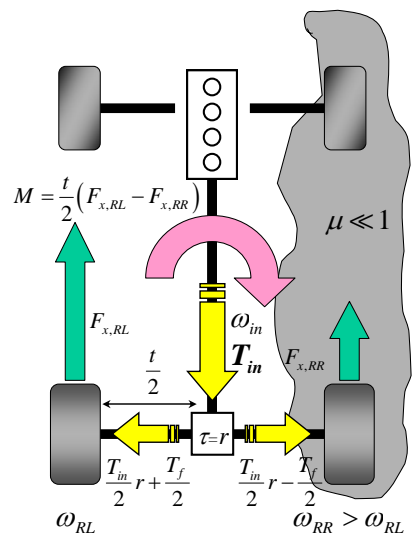

Fig. 12. Locking action of the differential in $\mu$-split.

The algorithm developed to evaluate the reference locking torque $T_{\text {free }}$, in vehicle start condition, adopts a feedback component based on a PI controller. This 
regulator gets as input the error between the actual difference of wheel speeds $\Delta V_{X, \text { ist }}$ and the threshold

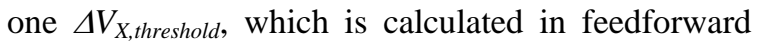
using a kinematical three wheel vehicle model (Fig. 13). Since this driving condition is characterized by very low sideslip angles, low speed and low lateral acceleration, a kinematical approach is accurate enough and it can be assumed as the reference. The model is used to calculate the maximum wheels speed difference $\Delta V_{X \text { threshold }}$ that the system can tolerate before actuating some locking torque. $\Delta V_{X, \text { threshold }}$ is mainly a function of steer angle and vehicle longitudinal speed according to (3), where $V_{X}$ is vehicle longitudinal speed coming from CAN bus, $\delta$ is the steer angle at wheel, $b$ is the car base and $t$ is the car rear track.

$$
\Delta V_{X, \operatorname{targ} e t}=\dot{\varphi} \cdot t=\frac{V_{X}}{\rho} t=\frac{V_{X} \cdot \tan (\delta)}{b} t
$$

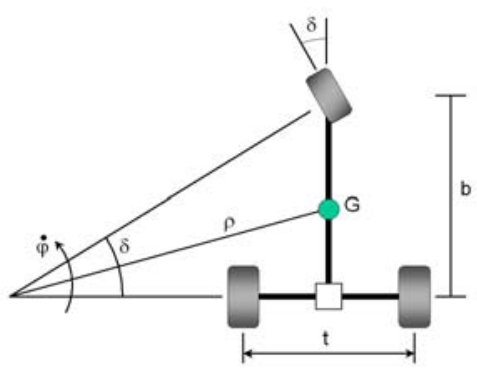

Fig. 13. Three wheel kinematical model.

When either the lateral acceleration either the longitudinal speed get so high that the kinematical model would not be accurate anymore, the control is smoothly transferred to the steady-state/power on algorithm.

\section{EXPERIMENTAL RESULTS}

When first release of the logic was developed, experimental testes have been executed. The aim was to verify and tune the logic in a demo car. In the figures below some result of the validation testes are reported. Steering wheel $\delta$, vehicle speed $V_{X}$, lateral acceleration $A_{Y}$ compared with the product of $V_{X}$ and YawRate, requested locking torque $T_{f, \text { ref }}$ and actual locking torque $T_{f}$ have been acquired for every manoeuvre. The first manoeuvre (Fig. 14 and Fig. 15) is a left-right sequence of turns executed in power on conditions. The steering angle varies from positive values to negative ones, while the speed is increasing. The $V_{X} \cdot$ YawRate compared with $A_{Y}$ shows that the car has a stable behaviour even if it approaches the limit of adherence. It's important to remember that a difference between $A_{Y}$ and $V_{X}$.YawRate means that the sideslip angle is increasing causing, at the limit, vehicle spin. In Fig. 15 system behaviour in terms of reference $T_{f, r e f}$ and applied $T_{f}$ torque, for the manoeuvre of Fig. 14, are reported. In Fig. 15 the effectiveness of the actuator layer control algorithm can be appreciate taking attention to the very little delay between the requested $T_{f, r e f}$ and the applied $T_{f}$ torque.
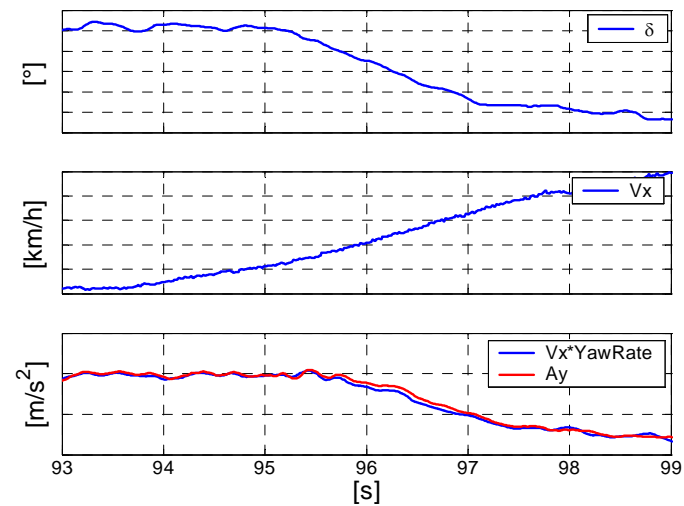

Fig. 14. Left-right turn. Steering angle (a), car speed (b), $A_{Y}$ vs. $V_{X}$ YawRate (c).

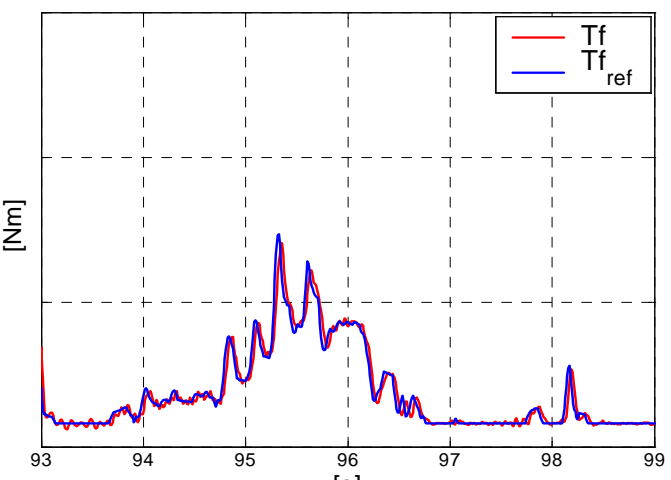

[s]

Fig. 15. Left-right turn. Actual $T_{f}$ and reference $T_{f, r e f}$ locking torque.

In Fig. 16 and Fig. 17 time histories for a power off in curve manoeuvre are shown. Also in this case the difference between $A_{Y}$ and $V_{X}$ YYawRate is very little even if the car is close to its lateral limit. Fig. 16c shows a very stable and smooth behaviour of the vehicle during a critical manoeuvre as the power off. In Fig. 17 requested $T_{f, \text { ref }}$ and actual $T_{f}$ locking torque time histories are shown: the system behaves even in a smoother way and actual locking torque is always close to its reference.
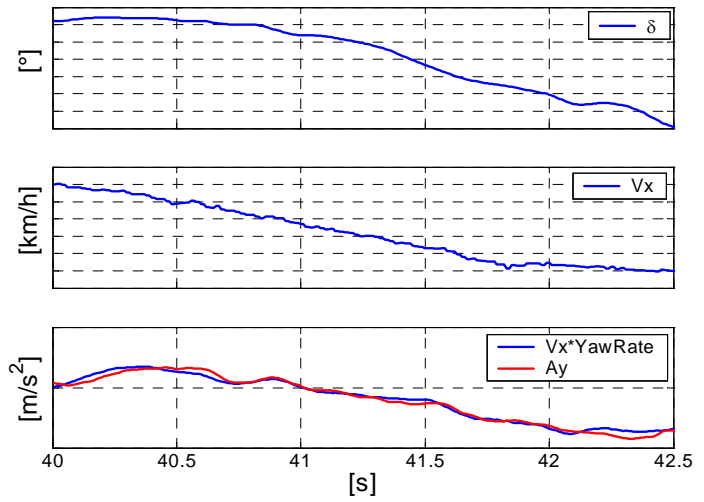

Fig. 16. Power off in turn. Steering angle (a), car speed (b), $A_{Y}$ vs. $V_{X} \cdot$ YawRate (c). 


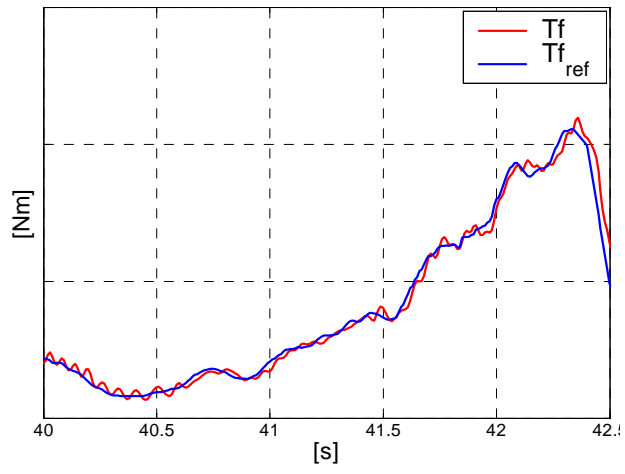

Fig. 17. Power off in turn. Actual $T_{f}$ and reference $T_{\text {fref }}$ locking torque.

In Fig. 18 and Fig. 19 it is compared the behaviour, in a power off manoeuvre, of a vehicle with free differential (Fig. 18) and with controlled one (Fig. 19). The vehicle with free differential shows an unstable behaviour: the YawRate increases (Fig. 18c) causing the reaction of the driver at time 18s.(Fig. 18a). The vehicle equipped with controlled differential is more stable and keeps the trajectory imposed by the driver, who has no reaction to the power off at time 21s.

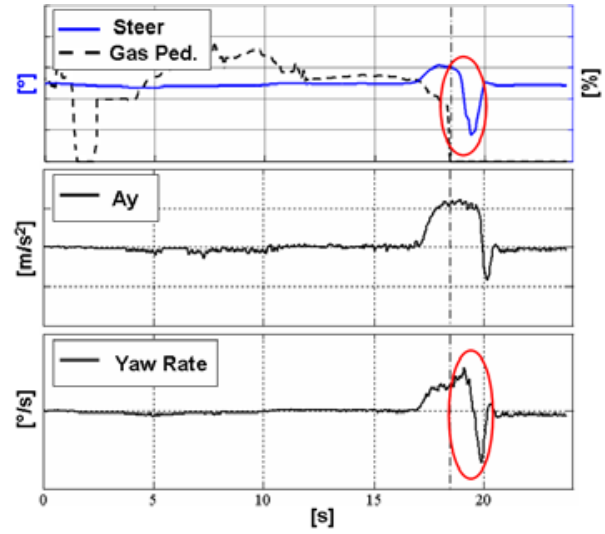

Fig. 18. Power off with free differential.

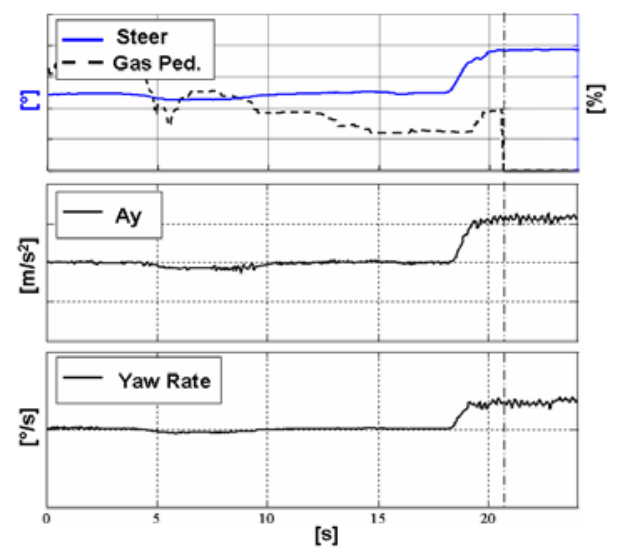

Fig. 19. Power off with controlled differential.

In Fig. 20 and Fig. 21 time histories for a stand-still start on $\mu$-split condition (ice/dry asphalt) are reported. Thanks to the controlled differential the car can accelerate at $0.3 \mathrm{~g}$ and the driver doesn't need to control any sever oversteering moment. In Fig. 21 the reference and actual locking torque are reported.
System dynamics is influenced by the inner frictions of the valve at very low torque values. Once reach normal usage values, the behaviour of the system is quite good. The car keeps a smooth and stable behaviour, while the locking torque improves tractions. The asymmetric distribution of the longitudinal contact forces causes the reaction of the driver, who smoothly adapts the steering wheel value.
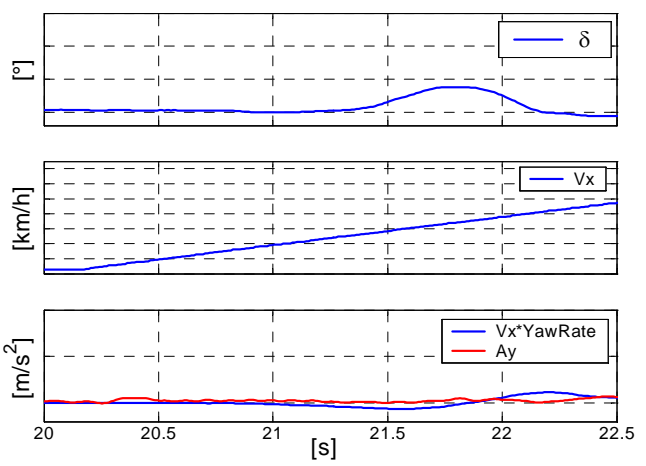

Fig. 20. Stand-still start on $\mu$-split condition. Steering angle (a), car speed (b), $A_{Y}$ vs. $V_{x} \cdot$ YawRate (c).

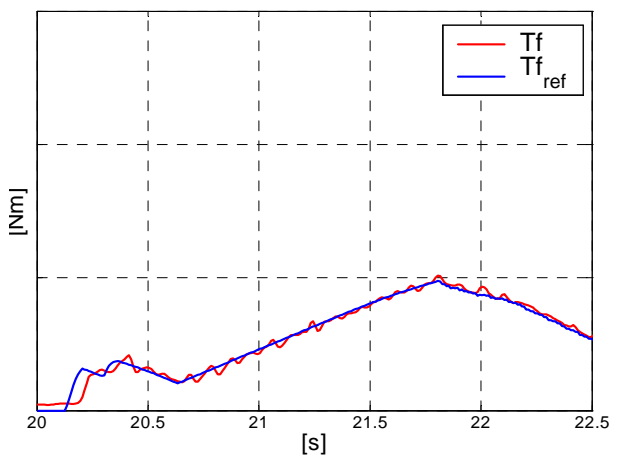

Fig. 21. Stand-still start on $\mu$-split condition. Actual $T_{f}$ and reference $T_{f, \text { ref }}$ locking torque.

\section{CONCLUSIONS}

In the two parts of the paper it has been presented the structure of the implemented control system and a deeper analysis of the developed Vehicle Dynamics control algorithms. The experimental results have shown the capability of the system in following its time-varying references (Part I) and in keeping a stable behaviour of the vehicle at its limits, while improving traction and absolute performance in term of driving feeling (Part II).

\section{REFERENCES}

Cheli, F., M. Giaramita, M. Pedrinelli and G. Sandoni, G. C. Travaglio, 2005, A new control strategy for a semi-active differential (Part I), Paper presented at 16th IFAC World Congress.

Pedrinelli, M. and A. Zorzutti, 2002, Sviluppo ed implementazione di una logica di controllo per il differenziale di una vettura sportiva, Degree Thesis A.A. 2001-2002 Politecnico di Milano (in Italian). 